\title{
EXPLOSION IN A DIFFUSIVE STRIP DUE TO A SOURCE WITH LOCAL AND NONLOCAL FEATURES
}

\author{
W. E. Olmstead and Catherine A. Roberts
}

\begin{abstract}
The possibility of a blow-up solution to the one-dimensional heat equation is examined for a nonlinear source that combines local and nonlocal features. The problem is analyzed by reduction to a pair of coupled nonlinear Volterra equations, for which existence as well as nonexistence through blow-up is investigated. Blow-up always occurs for the Neumann problem, whereas for the Dirichlet problem, blow-up depends upon the magnitude of certain parameters. An example is worked out that includes an asymptotic analysis to determine the growth rate near blow-up.
\end{abstract}

\section{Introduction}

We examine the explosive behavior of the solution to the heat equation in a onedimensional strip of finite width that contains a nonlinear heat source governed by both local and nonlocal effects. The problem will be treated for both the Neumann and Dirichlet type boundary conditions. Our investigation is carried out by converting the parabolic initial-boundary-value problem into a pair of nonlinear Volterra integral equations. This system of integral equations provides a description of the coupled relationship between the local and nonlocal effects.

Our analysis of the nonlinear Volterra equations will require an extension of the techniques developed in $[7,8,10]$. It will be shown that the Neumann problem always yields a blow-up solution; while the Dirichlet problem may or may not exhibit blow-up, depending upon the parameters of the problem.

Consider the temperature $v(x, t)$ in a finite strip as governed by the nonlinear heat equation,

$$
\frac{\partial v}{\partial t}(x, t)-\frac{\partial^{2} v}{\partial x^{2}}(x, t)=\delta(x-a) g[v(x, t)][V(t)]^{r}, \quad 0<x<\ell, \quad t>0,
$$

where $\delta(x-a)$ is the Dirac delta distribution with its singularity located at a fixed position $x=a, 0<a<\ell$, and

$$
V(t)=\|v(x, t)\|_{L_{1}}=\frac{1}{\ell} \int_{0}^{\ell}|v(x, t)| d x .
$$

Our interest here is in the blow-up properties of (1) with this nonlinear source which combines the localized contribution of $\delta(x-a) g[v(x, t)]$ with the global contribution of $[V(t)]^{r}$.

There has been considerable attention devoted to parabolic equations like (1) where the nonlinearity contains only $g[v(x, t)]$. See [5] for a review of that literature. More recently there has been work on localized nonlinearities of the form $\delta(x-a) g[v(x, t)]$ as

Received May 30, 1995, revised April 12, 1996.

1991 Mathematics Subject Classification: 35K60,45D05,45M05.

Key words and phrases: integral equations, blow-up solutions. 
used in $[2,7,9,10]$, or the more simple form of $g[v(a, t)]$ as used in [3]. A nonlinearity of the form $\|v(x, t)\|_{L_{1}} /[1-v(x, t)]$ has been used for a quenching problem in [4].

For (1)-(2), we impose the initial condition

$$
v(x, 0)=v_{0}(x) \geq 0, \quad 0 \leq x \leq \ell
$$

and either of the boundary conditions

$$
\begin{array}{rlrl}
\frac{\partial v}{\partial x}(0, t) & =\frac{\partial v}{\partial x}(\ell, t)=0 & & (\text { Neumann problem) } \\
v(0, t) & =v(\ell, t)=0 & & \text { or } \\
v(\text { Dirichlet problem). } &
\end{array}
$$

It is consistent with various explosion models to prescribe that the nonlinearity $g(v) V^{r}$ be such that,

$$
r>1, \quad g(v)>0, \quad g^{\prime}(v)>0, \quad g^{\prime \prime}(v)>0 \quad \text { for } v>0 .
$$

This behavior is compatible with the additional requirement that

$$
\int_{h_{0}}^{\infty} \frac{d v}{g(v) v^{r}}<\infty
$$

for some specific $h_{0}>0$.

As a first step in the analysis of (1)-(4), we introduce an integral representation of $v(x, t)$ in the form

$$
v(x, t)=\int_{0}^{t} \int_{0}^{\ell} G(x, t \mid \xi, s) \delta(\xi-a) g[v(\xi, s)][V(s)]^{r} d \xi d s+h(x, t),
$$

where $h(x, t)$ is given in terms of the initial data by

$$
h(x, t)=\int_{0}^{\ell} G(x, t \mid \xi, 0) v_{0}(\xi) d \xi .
$$

Here $G(x, t \mid \xi, s)$ is the Green's function for the linear heat equation with either the Neumann or Dirichlet type boundary conditions. Specifically, for the Neumann problem, $G=G_{N}$ where

$$
G_{N}(x, t \mid \xi, s)=H(t-s)\left\{\frac{1}{\ell}+\frac{2}{\ell} \sum_{n=1}^{\infty} \cos \left(\frac{n \pi \xi}{\ell}\right) \cos \left(\frac{n \pi x}{\ell}\right) e^{-\frac{n^{2} \pi^{2}}{\ell^{2}}(t-s)}\right\},
$$

while for the Dirichlet problem, $G=G_{D}$ where

$$
G_{D}(x, t \mid \xi, s)=H(t-s)\left\{\frac{2}{\ell} \sum_{n=1}^{\infty} \sin \left(\frac{n \pi \xi}{\ell}\right) \sin \left(\frac{n \pi x}{\ell}\right) e^{-\frac{n^{2} \pi^{2}}{\ell^{2}}(t-s)}\right\}
$$

where $H(t-s)$ is the Heaviside function.

Owing to the sifting property of the delta distribution, (7) becomes

$$
v(x, t)=\int_{0}^{t} G(x, t \mid a, s) g[v(a, s)][V(s)]^{r} d s+h(x, t), \quad t \geq 0 .
$$

In (11), it is seen that the determination of $v(x, t)$ depends upon the existence of $v(a, t)$ and $V(t)$. We investigate this issue by considering a pair of coupled Volterra equations for $v(a, t)$ and $V(t)$ derived from (11).

An evaluation of (11) at $x=a$ yields

$$
v(a, t)=\int_{0}^{t} G(a, t \mid a, s) g[v(a, s)][V(s)]^{r} d s+h(a, t), \quad t \geq 0 .
$$


From the conditions given for the boundary-value problem, it is clear that $v(x, t) \geq 0$ whenever it exists, and hence integration of (11) together with (2) yields

$$
V(t)=\int_{0}^{t}\left[\frac{1}{\ell} \int_{0}^{\ell} G(x, t \mid a, s) d x\right] g[v(a, s)][V(s)]^{r} d s+\frac{1}{\ell} \int_{0}^{\ell} h(x, t) d x, \quad t \geq 0 .
$$

Thus, the initial-boundary-value problem (1)-(4) has been reduced to the pair of nonlinear Volterra equations (12)-(13) for $v(a, t)$ and $V(t)$. For these integral equations, we note that the form of the nonlinearity is the same in each equation, while the kernels are different. Moreover, the explicit form of the kernels will depend upon whether the Neumann or Dirichlet problem is being considered. A system with some similarity to (12)-(13) was examined in [8], but there the kernels were the same while the nonlinearities were different in the two equations.

In our analysis to follow, we investigate the existence of a unique solution to the system (12)-(13), as well as non-existence through the occurrence of blow-up. The analysis will provide both upper and lower bounds on blow-up time. Finally, we will illustrate the application of these results to a specific example. In that example, we also will be able to determine explicitly the growth rates of $v(a, t)$ and $V(t)$ near blow-up by the application of asymptotic techniques.

\section{Existence of the solution}

Here we will examine some basic properties of the solution to the system (12)-(13) and establish both the existence and uniqueness of a bounded solution for $0 \leq t<t^{*}$. This will provide a lower bound $t^{*}$ for any blow-up that might occur.

To analyze (12)-(13), it is convenient to express the system as

$$
\begin{aligned}
& u(t)=\int_{0}^{t} k(t-s) g[u(s)+h(s)][\tilde{u}(s)+\tilde{h}(s)]^{r} d s, \quad t \geq 0 \\
& \tilde{u}(t)=\int_{0}^{t} \tilde{k}(t-s) g[u(s)+h(s)][\tilde{u}(s)+\tilde{h}(s)]^{r} d s, \quad t \geq 0
\end{aligned}
$$

where we define

$$
\begin{gathered}
v(a, t)-u(t) \equiv h(a, t) \equiv h(t) \\
V(t)-\tilde{u}(t) \equiv \frac{1}{\ell} \int_{0}^{\ell} h(x, t) d x \equiv \tilde{h}(t),
\end{gathered}
$$

and

$$
k(t-s)=G(a, t \mid a, s), \quad \tilde{k}(t-s)=\frac{1}{\ell} \int_{0}^{\ell} G(x, t \mid a, s) d x .
$$

The explicit forms of the kernels, $k(t-s)$ and $\tilde{k}(t-s)$, depend upon the choice of boundary conditions (4). For the Neumann problem, it follows from (9) that

$$
\begin{aligned}
& k(t-s)=k_{N}(t-s)=\frac{1}{\ell}+\frac{2}{\ell} \sum_{n=1}^{\infty} \cos ^{2}\left(\frac{n \pi a}{\ell}\right) e^{-\frac{n^{2} \pi^{2}}{\ell^{2}}(t-s)}, \\
& \tilde{k}(t-s)=\tilde{k}_{N}(t-s)=\frac{1}{\ell}
\end{aligned}
$$


while for the Dirichlet problem, it follows from (10) that

$$
\begin{aligned}
& k(t-s)=k_{D}(t-s)=\frac{2}{\ell} \sum_{n=1}^{\infty} \sin ^{2}\left(\frac{n \pi a}{\ell}\right) e^{-\frac{n^{2} \pi^{2}}{\ell^{2}}(t-s)}, \\
& \tilde{k}(t-s)=\tilde{k}_{D}(t-s)=\frac{2}{\pi \ell} \sum_{n=1}^{\infty}\left[\frac{1-(-1)^{n}}{n}\right] \sin \left(\frac{n \pi a}{\ell}\right) e^{-\frac{n^{2} \pi^{2}}{\ell^{2}}(t-s)} .
\end{aligned}
$$

There are certain properties of the kernels which are important in our analysis that follows. In the Appendix, it is shown for both the Neumann and Dirichlet cases that

$$
\begin{array}{ll}
k(t-s)>0, & k^{\prime}(t-s)<0, \\
\tilde{k}(t-s)>0, & \tilde{k}^{\prime}(t-s) \leq 0, \quad t \geq s,
\end{array}
$$

and

$$
k(t-s) \geq \tilde{k}(t-s), \quad t \geq s
$$

It also is convenient to impose two conditions on $h(t)$ and $\tilde{h}(t)$, both of which are determined by the initial data $v_{0}(x) \geq 0$. We will prescribe that

$$
\begin{array}{ll}
h^{\prime}(t) \geq 0, & 0<h_{0} \leq h(t) \leq h_{\infty}<\infty \\
\tilde{h}^{\prime}(t) \geq 0, & 0<h_{0} \leq \tilde{h}(t) \leq h_{\infty}<\infty .
\end{array}
$$

The positivity of the solutions to the system (14)-(15) is easily seen. Given the positivity of $h(t), \tilde{h}(t), k(t-s)$, and $\tilde{k}(t-s)$, along with the properties of the nonlinearities (5), it follows that

$$
u(t)>0, \quad \tilde{u}(t)>0 \quad \text { for } t>0
$$

whenever they exist.

To see that those functions also are increasing, we differentiate (14)-(15) to obtain

$$
\begin{aligned}
& u^{\prime}(t)=k(t) g[h(0)][\tilde{h}(0)]^{r}+\int_{0}^{t} k(t-s) R(s) d s, \\
& \tilde{u}^{\prime}(t)=\tilde{k}(t) g[h(0)][\tilde{h}(0)]^{r}+\int_{0}^{t} \tilde{k}(t-s) R(s) d s,
\end{aligned}
$$

where

$$
\begin{aligned}
R(s) \equiv g^{\prime} & {[u(s)+h(s)][\tilde{u}(s)+\tilde{h}(s)]^{r}\left[u^{\prime}(s)+h^{\prime}(s)\right] } \\
& +r g[u(s)+h(s)][\tilde{u}(s)+\tilde{h}(s)]^{r-1}\left[\tilde{u}^{\prime}(s)+\tilde{h}^{\prime}(s)\right] .
\end{aligned}
$$

A verification that (25)-(26) follow from differentiation of (14)-(15) is obtained from integration by parts together with the identity $\frac{\partial k}{\partial t}(t-s) \equiv-\frac{\partial k}{\partial s}(t-s)$. Given (24), then both $u^{\prime}(t)>0$ and $\tilde{u}^{\prime}(t)>0$ at least on some small interval $0<t<\bar{t}$. If either $u^{\prime}(\bar{t})=0$ or $\tilde{u}^{\prime}(\bar{t})=0$, then (25) would imply a contradiction since the right sides of both equations are positive. Thus

$$
u^{\prime}(t)>0, \quad \tilde{u}^{\prime}(t)>0, \quad 0<t<\infty,
$$

whenever they exist. We note that the conditions (23) that lead to the monotonic growth of $u(t)$ and $\hat{u}(t)$ are not essential for blow-up. 
To establish the existence of a unique solution to the system (14)-(15), it is convenient to introduce component notation. For $j=1,2$, we denote

$$
\begin{aligned}
& u_{j}(t): \quad u_{1}(t) \equiv u(t), \quad u_{2}(t) \equiv \tilde{u}(t), \\
& h_{j}(t): \quad h_{1}(t) \equiv h(t), \quad h_{2}(t) \equiv \tilde{h}(t), \\
& k_{j}(t): \quad k_{1}(t) \equiv k(t), \quad k_{2}(t) \equiv \tilde{k}(t) .
\end{aligned}
$$

Our analysis will utilize contraction mapping arguments based on the space of continuous functions that satisfy

$$
0 \leq u_{j}(t) \leq M<\infty, \quad j=1,2, \quad 0 \leq t<\hat{t},
$$

with the norm

$$
\left\|u_{j}\right\|=\sup _{0 \leq t<\hat{t}}\left\{\left|u_{1}(t)\right|+\left|u_{2}(t)\right|\right\} .
$$

The goal is to find the limitation on $\hat{t}$ under which the system operator of (14)-(15) provides a contraction mapping of this space into itself.

Given the properties of the kernels and the nonlinearities, it follows from (14)-(15) and (22) that

$$
0 \leq u_{j}(t) \leq g\left(M+h_{\infty}\right)\left(M+h_{\infty}\right)^{r} I(t), \quad j=1,2,
$$

where

$$
I(t) \equiv \max _{j=1,2} \int_{0}^{t} k_{j}(t-s) d s=\int_{0 .}^{t} k_{1}(t-s) d s .
$$

We note that $I^{\prime}(t)>0$, so that $I(t)$ is increasing. In order to insure that the upper bound of (29) holds, then $\hat{t}$ must be such that

$$
I(\hat{t}) \leq M\left[g\left(M+h_{\infty}\right)\left(M+h_{\infty}\right)^{r}\right]^{-1} .
$$

To establish the contraction property of the system operator $T_{j}, j=1,2$ defined by the right sides of (14)-(15), we consider two pairs of continuous functions $u_{j}(t)$ and $v_{j}(t), j=1,2$, that satisfy (29). Thus we find

$$
\begin{aligned}
& T_{1} u_{j}-T_{1} v_{j}=\int_{0}^{t} k_{1}(t-s)\left\{F\left[u_{j}(s)\right]-F\left[v_{j}(s)\right]\right\} d s \\
& T_{2} u_{j}-T_{2} v_{j}=\int_{0}^{t} k_{2}(t-s)\left\{F\left[u_{j}(s)\right]-F\left[v_{j}(s)\right]\right\} d s
\end{aligned}
$$

where

$$
\begin{aligned}
F\left[u_{j}(t)\right]-F\left[v_{j}(t)\right]=g[ & \left.u_{1}(t)+h_{1}(t)\right]\left[u_{2}(t)+h_{2}(t)\right]^{r} \\
& \quad-g\left[v_{1}(t)+h_{1}(t)\right]\left[v_{2}(t)+h_{2}(t)\right]^{r} \\
= & g^{\prime}\left[\theta_{1}(t)+h_{1}(t)\right]\left[\theta_{2}(t)+h_{2}(t)\right]^{r}\left[u_{1}(t)-v_{1}(t)\right] \\
& +r g\left[\theta_{1}(t)+h_{1}(t)\right]\left[\theta_{2}(t)+h_{2}(t)\right]^{r-1}\left[u_{2}(t)-v_{2}(t)\right],
\end{aligned}
$$

with the latter equality following from the mean value theorem by specifying that $\theta_{j}$ lies between $u_{j}$ and $v_{j}, j=1,2$. Given the properties of the kernels and nonlinearities, it follows that

$$
\begin{aligned}
& \left|T_{j} u_{i}-T_{j} v_{i}\right| \leq g^{\prime}\left(M+h_{\infty}\right)\left(M+h_{\infty}\right)^{r} \int_{0}^{t} k_{j}(t-s)\left|u_{1}(s)-v_{1}(s)\right| d s \\
& \quad+g\left(M+h_{\infty}\right) r\left(M+h_{\infty}\right)^{r-1} \int_{0}^{t} k_{j}(t-s)\left|u_{2}(s)-v_{2}(s)\right| d s, \quad j=1,2 .
\end{aligned}
$$


From (36), we obtain

$$
\left\|T_{j} u_{i}-T_{j} v_{i}\right\| \leq \alpha(M) I(t)\left\|u_{i}-v_{i}\right\|,
$$

where $I(t)$ is defined in (32) and

$$
\alpha(M)=2 \max \left\{g^{\prime}\left(M+h_{\infty}\right)\left(M+h_{\infty}\right)^{r}, g\left(M+h_{\infty}\right) r\left(M+h_{\infty}\right)^{r-1}\right\} .
$$

Thus, the system operator contracts whenever

$$
I(\hat{t})<[\alpha(M)]^{-1} \text {. }
$$

To determine a bounding value on $\hat{t}$ for which existence and uniqueness holds, it is convenient to define $t^{*}$ such that

$$
I\left(t^{*}\right)=\sup _{M \geq 0} I_{M}
$$

where for any $M \geq 0$,

$$
I_{M} \equiv \min \left\{M\left[g\left(M+h_{\infty}\right)\left(M+h_{\infty}\right)^{r}\right]^{-1},[\alpha(M)]^{-1}\right\} .
$$

This ensures that both (33) and (39) are fulfilled for $\hat{t}<t^{*}$.

Thus, we are able to conclude that there exists a unique continuous solution of (14)-(15) which is non-negative and increasing for $0 \leq t<t^{*}$. This $t^{*}$ represents a lower bound on any possible blow-up solution of (14)-(15).

\section{Nonexistence of the solution}

Here we determine conditions under which the solution of (14)-(15) can experience a blow-up. In particular, we will derive criteria that yield a $t^{* *}<\infty$ such that (14)-(15) cannot possess a continuous solution for $t \geq t^{* *}$.

We begin by assuming that (14)-(15) has a continuous solution for $0 \leq t \leq \hat{t}$. It then follows from the nonincreasing properties of the kernels (21) that

$$
\begin{aligned}
& u(t) \geq J(t) \equiv \int_{0}^{t} k(\hat{t}-s) g[u(s)+h(s)][\tilde{u}(s)+\tilde{h}(s)]^{r} d s, \\
& \tilde{u}(t) \geq \tilde{J}(t) \equiv \int_{0}^{t} \tilde{k}(\hat{t}-s) g[u(s)+h(s)][\tilde{u}(s)+\tilde{h}(s)]^{r} d s, \quad 0 \leq t \leq \hat{t} .
\end{aligned}
$$

Differentiation of the defined $J(t)$ and $\tilde{J}(t)$ gives

$$
\begin{aligned}
J^{\prime}(t) & =k(\hat{t}-t) g[u(t)+h(t)][\tilde{u}(t)+\tilde{h}(t)]^{r}, & & J(0)=0, \\
\tilde{J}^{\prime}(t) & =\tilde{k}(\hat{t}-t) g[u(t)+h(t)][\tilde{u}(t)+\tilde{h}(t)]^{r}, & \tilde{J}(0) & =0 .
\end{aligned}
$$

By utilizing (42)-(43) together with properties (22) and (23), it follows that

$$
\begin{array}{ll}
J^{\prime}(t) \geq \tilde{k}(\hat{t}-t) g\left[J(t)+h_{0}\right]\left[\tilde{J}(t)+h_{0}\right]^{r}, & J(0)=0, \\
\tilde{J}^{\prime}(t) \geq \tilde{k}(\hat{t}-t) g\left[J(t)+h_{0}\right]\left[\tilde{J}(t)+h_{0}\right]^{r}, & \tilde{J}(0)=0 .
\end{array}
$$

If we demonstrate blow-up for either $J(t)$ or $\tilde{J}(t)$, then (42)-(43) imply nonexistence for the corresponding solution of (14) and (15). To investigate blow-up for $J(t)$ or $\tilde{J}(t)$, we introduce a comparison problem

$$
\begin{aligned}
U^{\prime}(t) & =\tilde{k}(\hat{t}-t) g[U(t)][\tilde{U}(t)]^{r}, & & U(0)=h_{0}-\delta>0, \\
\tilde{U}^{\prime}(t) & =\tilde{k}(\hat{t}-t) g[U(t)][\tilde{U}(t)]^{r}, & & \tilde{U}(0)=h_{0}-\delta>0
\end{aligned}
$$


where $\delta>0$ is taken to be sufficiently small to insure the positivity of the initial conditions. To establish that

$$
W(t) \equiv J(t)+h_{0}-U(t)>0, \quad \tilde{W}(t) \equiv \tilde{J}(t)+h_{0}-\tilde{U}(t)>0,
$$

we combine (46)-(47) with (48)-(49) to obtain

$$
\begin{aligned}
& W^{\prime}(t) \geq \tilde{k}(\hat{t}-t)\{F[W(t)+U(t), \tilde{W}(t)+\tilde{U}(t)]-F[U(t), \tilde{U}(t)]\}, W(0)=\delta, \\
& \tilde{W}^{\prime}(t) \geq \tilde{k}(\hat{t}-t)\{F[W(t)+U(t), \tilde{W}(t)+\tilde{U}(t)]-F[U(t), \tilde{U}(t)]\}, \tilde{W}(0)=\delta,
\end{aligned}
$$

where

$$
F[U(t), \tilde{U}(t)]=g[U(t)][\tilde{U}(t)]^{r} .
$$

By use of the mean value theorem, it follows that

$$
\begin{array}{ll}
W^{\prime}(t) \geq \tilde{k}(\hat{t}-t)\{a(t) W(t)+\tilde{a}(t) \tilde{W}(t)\}, & W(0)=\delta, \\
\tilde{W}^{\prime}(t) \geq \tilde{k}(\hat{t}-t)\{a(t) W(t)+\tilde{a}(t) \tilde{W}(t)\}, & \tilde{W}(0)=\delta,
\end{array}
$$

where

$$
\begin{aligned}
& a(t) \equiv \frac{\partial F}{\partial U}[\theta(t), \tilde{\theta}(t)]=g^{\prime}\left[(\theta(t)][\tilde{\theta}(t)]^{r},\right. \\
& \tilde{a}(t) \equiv \frac{\partial F}{\partial \tilde{U}}[\theta(t), \tilde{\theta}(t)]=r g[\theta(t)][\tilde{\theta}(t)]^{r-1},
\end{aligned}
$$

and $\theta(t)$ lies between $W(t)+U(t)$ and $U(t)$, while $\tilde{\theta}(t)$ lies between $\tilde{W}(t)+\tilde{U}(t)$ and $\tilde{U}(t)$.

Integration of the differential inequalities (54)-(55) yields

$$
\begin{aligned}
& W(t) \geq \exp \left[\int_{0}^{t} \tilde{k}(\hat{t}-s) a(s) d s\right]\left\{\delta+\int_{0}^{t} \exp \left[-\int_{0}^{s} \tilde{k}(\hat{t}-\xi) a(\xi) d \xi\right] \tilde{W}(s) d s\right\}, \\
& \tilde{W}(t) \geq \exp \left[\int_{0}^{t} \tilde{k}(\hat{t}-s) \tilde{a}(s) d s\right]\left\{\delta+\int_{0}^{t} \exp \left[-\int_{0}^{s} \tilde{k}(\hat{t}-\xi) \tilde{a}(\xi) d \xi\right] W(s) d s\right\} .
\end{aligned}
$$

It is clear from (58)-(59) that $W(t)>0, \tilde{W}(t)>0$, and hence that (50) holds.

Having established (50), it then follows from (42)-(43) that

$$
u(t) \geq J(t) \geq[U(t)]_{\delta=0}-h_{0}, \quad \tilde{u}(t) \geq \tilde{J}(t) \geq[\tilde{U}(t)]_{\delta=0}-h_{0} .
$$

From this we conclude that the nonexistence by blow-up of either $U(t)$ or $\tilde{U}(t)$ implies the nonexistence by blow-up for $u(t)$ or $\tilde{u}(t)$, respectively.

The solution of the comparison problem (48)-(49) with $\delta=0$ is straightforward. The result, in implicit form, is given by

$$
U(t)=\tilde{U}(t), \quad \int_{h_{0}}^{U(t)} \frac{d z}{g(z) z^{r}}=\tilde{I}(t), \quad 0 \leq t \leq \hat{t}
$$

where

$$
\tilde{I}(t) \equiv \int_{0}^{t} \tilde{k}(t-s) d s .
$$

From (61), we can infer criteria for a blow-up in the functions of $U(t)$ and $\tilde{U}(t)$, and hence for $u(t)$ and $\tilde{u}(t)$. It is convenient to define

$$
\kappa \equiv \int_{h_{0}}^{\infty} \frac{d z}{g(z) z^{r}}
$$

which is given to be finite by (6). 
Thus, whenever $\kappa$ is in the range of $I(t)$, then (61) implies that both $U(t)$ and $\tilde{U}(t)$ experience blow-up. That is, if there exists a $t^{* *}<\infty$ such that

$$
\tilde{I}\left(t^{* *}\right)=\kappa,
$$

then

$$
U(t) \rightarrow \infty, \quad \tilde{U}(t) \rightarrow \infty \quad \text { as } t \rightarrow t^{* *} .
$$

In view of (65), the implication of (60) is that both $u(t)$ and $\tilde{u}(t)$ will experience blowup. Moreover, when either $u(t)$ or $\tilde{u}(t)$ ceases to exist by blow-up at some $t_{c} \leq t^{* *}$, then the form of (14)-(15) implies that the other also must cease to exist at the same $t_{c}$. Thus, whenever (64) is satisfied, then

$$
u(t) \rightarrow \infty, \quad \tilde{u}(t) \rightarrow \infty \quad \text { as } t \rightarrow t_{c},
$$

where

$$
t^{*} \leq t_{c} \leq t^{* *}
$$

\section{Contrast of the Neumann and Dirichlet problems}

Our results of Sections 2 and 3 indicate general criteria for the existence and nonexistence of a solution to the system (14)-(15) or, equivalently, (12)-(13). In brief, those results provide for the existence of a solution up to some $t^{*}$ as determined by (40), but deny existence beyond some $t^{* *}$ as determined by (64). The value of $t^{*}$ depends upon $I(t)$ as defined by (32), while the value of $t^{* *}$ depends upon $\tilde{I}(t)$ as defined by (62). The behavior of $I(t)$ and $\tilde{I}(t)$, which are expressed as integrals of the kernels $k(t-s)$ and $\tilde{k}(t-s)$, respectively, depend upon whether the Neumann or Dirichlet problem is being considered.

We can contrast the Neumann and Dirichlet problems by considering the explicit forms of $I(t)$ and $\tilde{I}(t)$ as determined from (19) and (20). We will find below that the Neumann problem always leads to blow-up; whereas, the Dirichlet problem may or may not experience blow-up, depending upon how near the localized feature of the source is placed to an endpoint. This type of effect was noted in [7] for a different nonlinear Volterra equation.

For the Neumann problem, it follows from (19) that

$$
I_{N}(t)=\int_{0}^{t} k_{N}(t-s) d s=\frac{t}{\ell}+\frac{2 \ell}{\pi^{2}} \sum_{n=1}^{\infty} \frac{1}{n^{2}} \cos ^{2}\left(\frac{n \pi a}{\ell}\right)\left(1-e^{-\frac{n^{2} \pi^{2} t}{\ell^{2}}}\right)
$$

and

$$
\tilde{I}_{N}(t)=\int_{0}^{t} \tilde{k}_{N}(t-s) d s=\frac{t}{\ell} .
$$

Using (68) in (39), it is clear that $I_{N}(t)$ always can be made sufficiently small to insure existence on some interval $0 \leq t<\hat{t}$. In contrast, however, by using (69) in (64), it is clear that $\tilde{I}_{N}(t)$ will eventually be large enough to imply blow-up.

For the Dirichlet problem, it follows from (20) that

$$
\begin{aligned}
I_{D}(t) & =\int_{0}^{t} k_{D}(t-s) d s=\frac{2 \ell}{\pi^{2}} \sum_{n=1}^{\infty} \frac{1}{n^{2}} \sin ^{2}\left(\frac{n \pi a}{\ell}\right)\left(1-e^{-\frac{n^{2} \pi^{2}}{\ell^{2}} t}\right) \\
& =\frac{a(\ell-a)}{\ell}-\frac{2 \ell}{\pi^{2}} \sum_{n=1}^{\infty} \frac{1}{n^{2}} \sin ^{2}\left(\frac{n \pi a}{\ell}\right) e^{-\frac{n^{2} \pi^{2}}{\ell^{2}} t}
\end{aligned}
$$


and

$$
\begin{aligned}
\tilde{I}_{D}(t) & =\int_{0}^{t} \tilde{k}_{D}(t-s) d s=\frac{2 \ell}{\pi^{3}} \sum_{n=1}^{\infty}\left[\frac{1-(-1)^{n}}{n^{3}}\right] \sin \left(\frac{n \pi a}{\ell}\right)\left(1-e^{-\frac{n^{2} \pi^{2}}{\ell^{2}} t}\right) \\
& =\frac{a(\ell-a)}{2 \ell}-\frac{2 \ell}{\pi^{3}} \sum_{n=1}^{\infty}\left[\frac{1-(-1)^{n}}{n^{3}}\right] \sin \left(\frac{n \pi a}{\ell}\right) e^{-\frac{n^{2} \pi^{2}}{\ell^{2}} t} .
\end{aligned}
$$

Using (70) in (39), it is clear that $I_{D}(t)$ always can be made sufficiently small to insure existence for all $t>0$. In particular, the requirement that

$$
I_{D}(\infty)=\frac{a(\ell-a)}{\ell}<[\alpha(M)]^{-1}
$$

is adequate to insure existence for all $t>0$ and, hence, no blow-up. We always can satisfy (72) by placing the source singularity near enough to a boundary (i.e., $a \rightarrow 0$ or $a \rightarrow \ell)$.

On the other hand, by using (71) in (64), it is clear that it may be possible to fulfill the criterion for blow-up. In particular, if the parameters are such that

$$
\frac{a(\ell-a)}{2 \ell}>\kappa
$$

then a blow-up will occur since there must be some sufficiently large $t^{* *}$ such that $\tilde{I}_{D}\left(t^{* *}\right)=\kappa$. The abililty to satisfy (73) will depend upon the length of the domain as well as the placement of the nonlinear source.

A physical explanation of this disparate behavior between the two types of boundary conditions is that when the ends of the domain are insulated (Neumann problem), no heat can escape and hence the energy from the nonlinear source eventually reaches a level which is sufficient to produce blow-up. In contrast, when the ends of the domain are maintained at constant temperature (Dirichlet problem), considerable heat can be lost. Then, the occurrence of blow-up will depend upon the amount of energy that escapes through the boundaries. This loss can be enhanced by placing the source close to one of the cold boundaries.

\section{An example including growth rates}

To illustrate the results of Sections 2 and 3, we will consider a specific example of a Neumann problem. For this example we will obtain the bounds on the blow-up time. Furthermore, we will use asymptotic methods to determine the growth rates of the solution near blow-up.

We consider (14)-(15) for

$$
g(v)=v^{m}, \quad 1<m<2 .
$$

Given the form of (74), blow-up will actually occur for all $m>1$; however, our asymptotic analysis to determine the growth rate is only valid for $m<2$. For simplicity, we take

$$
h(t) \equiv 1, \quad \tilde{h}(t) \equiv 1, \quad t \geq 0 .
$$

For the Neumann problem, (14)-(15) then take the form

$$
\begin{aligned}
& u(t)=\int_{0}^{t} k_{N}(t-s)[u(s)+1]^{m}[\tilde{u}(s)+1]^{r} d s, \quad t \geq 0, \\
& \tilde{u}(t)=\frac{1}{\ell} \int_{0}^{t}[u(s)+1]^{m}[\tilde{u}(s)+1]^{r} d s, \quad t \geq 0,
\end{aligned}
$$


where $k_{N}(t-s)$ is given by (19).

In the case of the Neumann problem, blow-up always occurs. We can use the results of Sections 2 and 3 to find the lower and upper bounds, respectively, on the blow-up time.

For the lower bound, we first calculate from (38) that

$$
\begin{aligned}
\alpha(M) & =2 \max \left\{m(M+1)^{m-1}(M+1)^{r}, r(M+1)^{m}(M+1)^{r-1}\right\} \\
& =2 \gamma(M+1)^{m+r-1}, \quad \gamma=\max \{m, r\},
\end{aligned}
$$

and then, from (41) that

$$
I_{M}=\min \left\{M(M+1)^{-m-r},(2 \gamma)^{-1}(M+1)^{-m-r+1}\right\} .
$$

To obtain the right side of (40), it is noted that $I_{M}$ has its maximum at the intersection of the two functions that define it, and hence

$$
M=\frac{1}{2 \gamma-1}, \quad \sup _{M \geq 0} I_{M}=\frac{(2 \gamma-1)^{m+r-1}}{(2 \gamma)^{m+r}} .
$$

The left side of (40) is found from the integration of $k_{N}(t-s)$. Consequently, $t^{*}$ is determined from

$$
\frac{\ell t^{*}}{2}+\frac{2 \ell}{\pi^{2}} \sum_{n=1}^{\infty} \frac{1}{n^{2}} \cos ^{2}\left(\frac{n \pi a}{\ell}\right)\left(1-e^{-\frac{n^{2} \pi^{2}}{\ell^{2}} t^{*}}\right)=\frac{(2 \gamma-1)^{m+r-1}}{(2 \gamma)^{m+r}} .
$$

For the upper bound, we first calculate from (63) that

$$
\kappa=\int_{1}^{\infty} \frac{d z}{z^{m+r}}=\frac{1}{m+r-1} .
$$

The left side of (64) is found from the integration of $\tilde{k}_{N}(t-s)=\ell^{-1}$. Consequently, $t^{* *}$ is determined to be

$$
t^{* *}=\frac{\ell}{(m+r-1)} .
$$

Since (83) always is fulfilled for some $t^{* *}<\infty$, then there will be blow-up as $t \rightarrow t_{c}$ where $t^{*} \leq t_{c} \leq t^{* *}$.

To determine the growth rates near blow-up for $u(t)$ and $\tilde{u}(t)$ in this example, we will employ an asymptotic analysis similar to that used in [6-10] which, in turn, is based upon techniques developed in [1]. This analysis does not determine the explicit value of $t_{c}$, but rather demonstrates a self-consistent satisfaction of (76)-(77) near blow-up. For convenience, we introduce the transformation

$$
\eta=\left(t_{c}-t\right)^{-1}-\eta_{0}, \quad \eta_{0}=\left(t_{c}\right)^{-1}, \quad w(\eta)=u(t), \quad \tilde{u}(\eta)=\tilde{u}(t),
$$

whereupon the blow-up as $t \rightarrow t_{c}$, as expressed in (66), takes the form

$$
w(\eta) \rightarrow \infty, \quad \tilde{w}(\eta) \rightarrow \infty \quad \text { as } \eta \rightarrow \infty .
$$

The transformation (84) converts (76)-(77) to

$$
\begin{gathered}
w(\eta)=\int_{0}^{\eta} k_{N}\left\{(\eta-\sigma)\left[\left(\sigma+\eta_{0}\right)\left(\eta+\eta_{0}\right)\right]^{-1}\right\} \Phi(\sigma) d \sigma, \quad \eta \geq 0 \\
\tilde{w}(\eta)=\frac{1}{\ell} \int_{0}^{\eta} \Phi(\sigma) d \sigma, \quad \eta \geq 0
\end{gathered}
$$

where

$$
\Phi(\eta) \equiv\left(\eta+\eta_{0}\right)^{-2}[w(\eta)+1]^{m}[\tilde{w}(\eta)+1]^{r} .
$$


For the analysis of (86)-(87) as $\eta \rightarrow \infty$, it is convenient to replace (86) by an appropriate asymptotic form as derived in [9]. It was shown there that

$$
w(\eta) \sim \eta^{\frac{1}{2}} \int_{0}^{\eta} \frac{\left(\sigma+\eta_{0}\right)^{\frac{1}{2}} \Phi(\sigma)}{2[\pi(\eta-\sigma)]^{\frac{1}{2}}} d \sigma=\eta^{\frac{1}{2}} \ell \int_{0}^{\eta} \frac{\left(\sigma+\eta_{0}\right)^{\frac{1}{2}} \tilde{w}^{\prime}(\sigma)}{2[\pi(\eta-\sigma)]^{\frac{1}{2}}} d \sigma, \quad \eta \rightarrow \infty,
$$

where we also have introduced the equivalent form of (87) as

$$
\tilde{w}^{\prime}(\eta)=\frac{1}{\ell} \Phi(\eta)
$$

To determine the asymptotic solution of (89)-(90), we consider

$$
w(\eta) \sim A \eta^{b}, \quad \tilde{w}(\eta) \sim \tilde{A} \eta^{c} \quad \text { as } \eta \rightarrow \infty .
$$

Then, by the application of results given in $[1,6]$ for the asymptotic behavior of Riemann-Liouville fractional integrals, (89) takes the form

$$
A \eta^{b} \sim \ell \tilde{A} c \frac{\Gamma\left(c+\frac{1}{2}\right)}{\Gamma(c+1)} \eta^{c+\frac{1}{2}}, \quad c \leq \frac{1}{2}, \quad \eta \rightarrow \infty
$$

while (90) implies

$$
\tilde{A} c \eta^{c-1} \sim \frac{A^{m} \tilde{A}^{r}}{\ell} \eta^{b m+c r-2}, \quad \eta \rightarrow \infty .
$$

The simultaneous satisfaction of (92)-(93) yields

$$
\begin{gathered}
b=\frac{r+1}{2(m+r-1)}, \quad c=\frac{2-m}{2(m+r-1)}, \\
A=\left[\frac{\Gamma(c+1)}{\Gamma\left(c+\frac{1}{2}\right)}\right]^{\frac{1-r}{m+r-1}}(c \ell)^{\frac{r}{m+r-1}}, \quad \tilde{A}=\left[\frac{\Gamma(c+1)}{\Gamma\left(c+\frac{1}{2}\right)}\right]^{\frac{m}{m+r-1}}(c \ell)^{\frac{1-m}{m+r-1}} .
\end{gathered}
$$

Thus, for (76)-(77), we have determined the growth rates near blow-up, that is,

$$
u(t) \sim A\left(t_{c}-t\right)^{-\frac{r+1}{2(m+r-1)}}, \quad \tilde{u}(t) \sim \tilde{A}\left(t_{c}-t\right)^{-\frac{2-m}{2(m+r-1)}} \quad \text { as } t \rightarrow t_{c}<\infty,
$$

where $A$ and $\tilde{A}$ are given by (95).

Acknowledgement. The research of the first author was supported by NSF Grant DMS-9401016, while that of the second author was supported by NSF Grant DMS9510082.

\section{Appendix}

In Sections 2 and 3, certain properties of the kernels, as expressed in (21)-(22), are employed. The validity of those properties, for both the Neumann and Dirichlet cases, is established here. Toward that goal, it is convenient to consider the linear initialboundary-value problems,

$$
\begin{gathered}
\left(\frac{\partial}{\partial t}-\frac{\partial^{2}}{\partial x^{2}}\right) w(x, t ; \xi)=0, \quad 0<x<\ell, \quad t>0, \\
w(x, 0 ; \xi)=\delta(x-\xi), \quad 0 \leq x \leq \ell, \quad 0<\xi<\ell, \\
\frac{\partial w}{\partial x}(0, t ; \xi)=\frac{\partial w}{\partial x}(\ell, t ; \xi)=0 \quad \text { or } \quad w(0, t ; \xi)=w(\ell, t ; \xi)=0, \quad t>0,
\end{gathered}
$$


and

$$
\begin{gathered}
\left(\frac{\partial}{\partial t}-\frac{\partial^{2}}{\partial x^{2}}\right) \tilde{w}(x, t)=0, \quad 0<x<\ell, \quad t>0, \\
\tilde{w}(x, 0)=\frac{1}{\ell}, \quad 0 \leq x \leq \ell, \\
\frac{\partial \tilde{w}}{\partial x}(0, t)=\frac{\partial \tilde{w}}{\partial x}(\ell, t)=0 \quad \text { or } \quad \tilde{w}(0, t)=\tilde{w}(\ell, t)=0, \quad t>0 .
\end{gathered}
$$

For these problems, we let $w_{N}(x, t ; \xi)$ and $\tilde{w}_{N}(x, t)$ denote the solutions for the Neumann boundary conditions, while $w_{D}(x, t ; \xi)$ and $\tilde{w}_{D}(x, t)$ denote the solutions for the Dirichlet boundary conditions.

The solutions to the above problems are obtainable by elementary methods. They can be expressed as

$$
\begin{aligned}
w_{N}(x, t ; \xi) & =\frac{1}{\ell}+\frac{2}{\ell} \sum_{n=1}^{\infty} \cos \left(\frac{n \pi \xi}{\ell}\right) \cos \left(\frac{n \pi x}{\ell}\right) e^{-\frac{n^{2} \pi^{2}}{\ell^{2}} t} \\
w_{D}(x, t ; \xi) & =\frac{2}{\ell} \sum_{n=1}^{\infty} \sin \left(\frac{n \pi \xi}{\ell}\right) \sin \left(\frac{n \pi x}{\ell}\right) e^{-\frac{n^{2} \pi^{2}}{\ell^{2}} t} \\
\tilde{w}_{N}(x, t) & =\frac{1}{\ell} \\
\tilde{w}_{D}(x, t) & =\frac{2}{\pi \ell} \sum_{n=1}^{\infty}\left[\frac{1-(-1)^{n}}{n}\right] \sin \left(\frac{n \pi x}{\ell}\right) e^{-\frac{n^{2} \pi 2}{\ell^{2}} t}
\end{aligned}
$$

A comparison of (103)-(106) with (19)-(20), reveals that

$$
k_{N}(t-s)=w_{N}(a, t-s ; a), \quad \tilde{k}_{N}(t-s)=\tilde{w}_{N}(a, t-s),
$$

and

$$
k_{D}(t-s)=w_{D}(a, t-s ; a), \quad \tilde{k}_{D}(t-s)=\tilde{w}_{D}(a, t-s) .
$$

It then follows that

$$
k_{N}(t-s)>0, \quad \tilde{k}_{N}(t-s)>0, \quad k_{D}(t-s)>0, \quad \tilde{k}_{D}(t-s)>0,
$$

where all but the last are clear from (103)-(105). The last becomes apparent when it is noted from (100)-(102) that the maximum principle for the heat equation gives $\tilde{w}_{D}(x, t)>0,0<x<\ell, t \geq 0$.

It also follows that

$$
k_{N}^{\prime}(t-s) \geq 0, \quad \tilde{k}_{N}^{\prime}(t-s)=0, \quad k_{D}^{\prime}(t-s) \geq 0, \quad \tilde{k}_{D}^{\prime}(t-s) \geq 0 .
$$

where again all but the last are clear from (103)-(105). The last also becomes apparent when it is noted from (100)-(102) that $\tilde{w}_{D}(a, t)$ must be a decreasing function of $t$ by virtue of the maximum principle.

The property that

$$
k_{N}(t-s) \geq \tilde{k}_{N}(t-s),
$$

follows from (103) and (105) by observing that $w_{N}(a, t ; a) \geq \tilde{w}_{N}(a, t)$.

To establish that

$$
k_{D}(t-s) \geq \tilde{k}_{D}(t-s)
$$


it is first noted from (104) and (106) that

$$
\tilde{w}_{D}(a, t-s)=\frac{1}{\ell} \int_{0}^{\ell} w_{D}(a, t-s ; \xi) d \xi .
$$

From this, we obtain

$$
\tilde{w}_{D}(a, t-s) \leq \max _{0 \leq \xi \leq \ell} w_{D}(a, t-s ; \xi)=w_{D}(a, t-s ; a),
$$

where the equality follows by noting that, for any $t>0, w(x, t ; \xi)$ achieves the largest value at $x=\xi$. From (108), we see that (114) validates (112).

\section{References}

1. N. Bleistein and R. A. Handelsman, Asymptotic Expansion of Integrals, Holt, Rinehardt, and Winston, New York, 1975.

2. J. M. Chadam, A. Peirce, and H.-M. Yin, The blowup property of solutions to some diffusion equations with localized nonlinear reactions, J. Math. Anal. Appl. 169 (1992), 313-328.

3. J. M. Chadam and H.-M. Yin, A diffusion equation with localized chemical reactions, Proc. Edinburgh Math. Soc. 37 (1993), 101-118.

4. K. Deng, Dynamical behavior of solutions of a semilinear heat equation with nonlocal singularity, SIAM J. Math. Anal. 26 (1995), 98-111.

5. H. A. Levine, The role of critical exponents in blow-up theorems, SIAM Rev. 32 (1990), 262-288.

6. W. E. Olmstead and R. A. Handelsman, Asymptotic solution to a class of nonlinear Volterra integral equations II, SIAM J. Appl. Math. 30 (1976), 180-189.

7. W. E. Olmstead and C. A. Roberts, Explosion in a diffusive strip due to a concentrated nonlinear source, Methods Appl. Anal. 1 (1994), 434-445.

8. W. E. Olmstead, C. A. Roberts, and K. Deng, Coupled Volterra equations with blow-up solutions, J. Integral Eqn. Appl. 7 (1995), 499-516.

9. C. A. Roberts and W. E. Olmstead, Growth rates for blow-up solutions of nonlinear Volterra equations, Quart. Appl. Math. 54 (1996), 153-160.

10. C. A. Roberts, W. E. Olmstead, and D. G. Lasseigne, Volterra equations which model explosion in a diffusive medium, J. Integral Eqn. Appl. 5 (1993), 531-546.

Department of Engineering Sciences and Applied Mathematics, Northwestern University, EvANSTON, IL 60208

Department of Mathematics, Northern Arizona University, Flagstaff, AZ 86011 